\title{
El liderazgo inclusivo como estrategia para evitar el abandono escolar: opinión de las familias ${ }^{1}$
}

\section{Inclusive leadership as a strategy to avoid school leaving: opinion of families}

\author{
EMilio Crisol MOYA² \\ ecrisol@ugr.es \\ María Asunción Romero López \\ romerol@ugr.es \\ Universidad de Granada, España
}

\begin{abstract}
Resumen:
Hablar de inclusión a nivel de centro inevitablemente "lleva consigo hablar de una relación activa y positiva entre familia y escuela" (Simón, Giné y Echeita, 2016, p. 26). En esta línea, se ha constatado la importancia de la participación de las familias en los centros escolares y su consecuente mejora en el rendimiento académico del alumnado; considerándose un factor clave en la prevención del abandono escolar. Este artículo se centra en determinar la perspectiva de las familias respecto al liderazgo inclusivo desarrollado por los equipos directivos, tales como la apertura del centro a la comunidad y el entorno, y acciones para impulsar la participación, atender la diversidad, prevenir y gestionar los conflictos en los centros en pro de un aprendizaje para todos. Se trata de una investigación cuantitativa
\end{abstract}

\begin{abstract}
:
Talking about inclusion at a school level inevitably "involves an active and positive relationship between families and schools" (Simón, Giné y Echeita, 2016, p. 26). Along these lines, the importance of family participation in schools has been reported to lead to improvements in students' academic performance, which, in turn, stands out as a key factor in the prevention of early school leaving. This article aims to determine the views of families on inclusive leadership measures developed by management teams, such as (i) opening the center to the community and the environment; and (ii) actions to encourage participation, address diversity, and prevent and manage conflict in centers so that learning can be for everybody. This is a descriptive quantitative investigation. In this sense, our sample is composed of
\end{abstract}

1 Este artículo se ha realizado dentro del Proyecto I+D+i. Identidad de la dirección escolar: liderazgo, formación y profesionalización (EDU2016-78191-P) financiado por el Ministerio de Ciencia e Innovación.

2 Dirección para correspondencia (correspondence address):

Emilio Crisol Moya. Universidad de Granada. Departamento de Didáctica y Organización Escolar. Facultad de Ciencias de la Educación. Campus Universitario de Cartuja C.P. 18071 Granada (España). 
El liderazgo inclusivo como estrategia para evitar el abandono escolar: opinión de las familias

Emilio Crisol Moya y María Asunción Romero López

de carácter descriptivo; la muestra estuvo compuesta por familiares de alumnos $(\mathrm{N}=150)$. Utilizamos como herramienta el cuestionario: "Liderando la Educación Inclusiva" (LEI-Q) (León et al., 2016). Los resultados muestran que las familias consideran que sus equipos directivos han implantado "sustancialmente" la mayor parte de las acciones que contribuyen a que sus centros sean inclusivos, promoviendo estrategias que reduzcan el abandono escolar. De esta manera se hace notable la necesidad de involucrar a las familias, para garantizar el éxito educativo y estimular a finalizar los estudios obligatorios y a continuar con una formación posterior.

\section{Palabras clave:}

Abandono escolar; familias; equipo directivo; liderazgo inclusivo; enseñanza obligatoria. students' relatives $(N=150)$, who were asked to complete the questionnaire "Leading Inclusive Education" (LEI-Q) (León et al., 2016). This research is an ex post-facto cross-sectional investigation of a descriptive nature. The results obtained indicate that families perceive the efforts made by school management teams to foster inclusive schools, which, in turn, promotes strategies that reduce school dropout. In this way, it is essential to involve families in order to guarantee educational success and stimulate students' completion of compulsory education and to continue their further training.

\section{Key words:}

Early school leaving; families; school management team; inclusive leadership; compulsory education.

\section{Résumé :}

Parler de l'inclusion au niveau du centre «implique inévitablement de parler d'une relation active et positive entre la famille et l'école» (Simón, Giné et Echeita, 2016, p. 26). Dans ce sens, l'importance de la participation des familles à l'école et de l'amélioration qui en résulte dans les résultats scolaires des élèves a été vérifiée; considéré comme un facteur clé dans la prévention du décrochage scolaire. Cet article cherche à déterminer le point de vue des familles concernant le leadership inclusif développé par les équipes de gestion, telles que l'ouverture du centre à la communauté et à l'environnement, et les actions pour promouvoir la participation, aborder la diversité, prévenir et gérer les conflits dans des centres d'apprentissage pour tous. II s'agit d'une enquête quantitative descriptive; l'échantillon étant composé de membres de la famille des étudiants $(N=$ 150). Nous utilisons comme outil le questionnaire: «Leading Inclusive Education» (LEI-Q) (León et al., 2016). Les résultats montrent que les familles considèrent que leurs équipes de gestion ont «substantiellement» mis en œuvre la plupart des actions qui contribuent à rendre leurs centres inclusifs, en promouvant des stratégies qui réduisent le décrochage scolaire. De cette manière, la nécessité d'impliquer les familles est notable, afin de garantir la réussite scolaire et de stimuler l'achèvement des études obligatoires et de poursuivre la formation ultérieure.

\section{Mots-clés:}

Abandon scolaire; familles; équipe de direction; leadership inclusif; enseignement obligatoire.

\footnotetext{
Fecha de recepción: 15-02-2020

Fecha de aceptación: 06-03-2020
} 


\section{Introducción}

Conseguir una educación de calidad en una sociedad cada vez más diversa hace necesario propiciar a nivel de centro un liderazgo inclusivo. Hablar de inclusión a nivel de centro, Ileva consigo hablar de una relación activa y positiva entre familia y escuela. Ambos, familia y escuela, son expertos y modelos educativos fundamentales de quienes quieren aprender. De ahí la importancia y necesidad de colaborar juntos si quieren que la tarea educativa alcance todo su potencial, especialmente cuando la literatura científica ha dejado constancia de que dicha colaboración contribuye a la mejora del rendimiento y motivación escolar; reduce el abandono escolar (Álvarez y Martínez, 2016); mejora el clima del centro y disminuye los problemas de convivencia (Cross y Barnes, 2014; Roberts, 2018); y promueve la democratización y dinamismo de la escuela (Batanova y Loukas, 2014; Roberts, 2018). Para aproximarnos a la inclusión, "hemos de concebirla como un proceso continuo de deconstrucción y reconstrucción de la cultura escolar, de las políticas y de las prácticas de cada centro" (Moreno y León, 2017, p. 212), con el fin de descubrir las barreras que imposibilitan el desarrollo del aprendizaje en el alumnado así como su participación (Ainscow, Booth y Dyson, 2006; Booth y Ainscow, 2000; León, 2011). De esta forma, como indica Echeita (2012) "no podemos entender la inclusión como una característica minoritaria del sistema educativo, sino como parte esencial para la construcción del sistema social deseado" (citado en Moreno y León, 2017, p. 212). Para ello, es necesario, como indican Escudero y Martínez (2011, p. 88), “(...) que la educación inclusiva no sea un enfoque inspirado en opciones caritativas y particulares, sino en imperativos morales y de justicia social".

El liderazgo, en el ámbito escolar, se considera uno de los factores que ayuda a disminuir el abandono escolar; según el informe TALIS de la OCDE (2018), después de la práctica docente en el aula, es el segundo factor más influyente e+n los logros del alumnado; aunque su influencia sea indirecta y se ejerce mediante una adecuada organización escolar y el desarrollo de una enseñanza de calidad, que a su vez potencia la calidad del profesorado (Bolívar, 2010).

La suma de estos dos conceptos previos, da lugar al liderazgo inclusivo (León, Crisol y Arrebola, 2018), que como indican Booth y Ainscow (2000), debe estar enfocado principalmente a conseguir una "cultura in- 
El liderazgo inclusivo como estrategia para evitar el abandono escolar: opinión de las familias

Emilio Crisol Moya y María Asunción Romero López

clusiva, la creación de una comunidad escolar en la que cada miembro es valorado y se fomente su nivel de logro y además se caracterice por ser acogedora, segura, colaboradora y estimulante" (citado en Moreno y León, 2017, p. 213).

El liderazgo inclusivo ejerce una influencia positiva en la práctica del profesorado (Angelides, Antoniu y Chalambous, 2010), en la calidad de los aprendizajes y participación del alumnado (Ainscow y Kaplan, 2005), así como en la mejora de los centros y en la creación una cultura inclusiva (Muijs, 2006; Ryan, 2006). Por ello, es necesario "profundizar en las características y prácticas que desempeñan los equipos directivos para alcanzar estas metas, en la formación necesaria para ejercerlas y en las condiciones/factores que confluyen para que se desempeñen adecuadamente las mismas" (León, Crisol y Moreno, 2018, p. 23).

En esta línea, se ha constatado la importancia de la participación de las familias en los centros escolares y su consecuente mejora en el rendimiento académico del alumnado, constatándose como un factor preventivo del abandono escolar.

De acuerdo con las conclusiones extraídas de investigaciones relevantes sobre el liderazgo inclusivo, algunas de las tareas y/o funciones desempeñadas por el equipo directivo para cumplir las metas de un centro inclusivo podemos agruparlas (León, Crisol y Arrebola, 2018) en: apertura a la comunidad (a través del planteamiento de iniciativas para la apertura del centro hacia el entorno y la familia, así como medidas dirigidas al éxito de todos e igualdad de oportunidades); el centro educativo como espacio inclusivo (emprende acciones para impulsar la participación, atender a la diversidad, y prevenir y gestionar los conflictos en la institución).

En este artículo abordamos el liderazgo como forma de potenciar la inclusión en los centros educativos (León, 2012), pues existen evidencias de que este es una pieza clave en el cambio y mejora de los mismos (Bolívar, López y Murillo, 2013), y el segundo factor en la reducción del abandono escolar (TALIS, 2018). Por ello, consideramos de gran relevancia profundizar en el grado en que la dirección de los centros educativos lleva a cabo tareas que promueven la inclusión de la comunidad y en concreto de las familias, y determinar qué características, tanto de los equipos directivos como de los centros, correlacionan positivamente con las mismas. 


\section{Marco teórico}

El abandono escolar constituye uno de los grandes problemas para todos los sistemas educativos a nivel mundial en la actualidad. El abandono escolar, al igual que el éxito escolar, está determinado por múltiples componentes o factores y causas, considerándose entre estas: sociales, económicas y educativas (Roca, 2010). En cuanto a los componentes del abandono escolar, De Witte, Cabus, Thyssen, Groot y Brink (2014) los clasifican en factores del alumnado, familiares, de la escuela y de la comunidad. Aunque Lozano (2012) destaca tres tipos de factores que intervienen en el abandono escolar: el familiar, el académico y el personal (los propios del alumnado). En este sentido, Rittacco y Amores (2016) enfatizan que el alumnado no solo fracasa "por dificultades de aprendizaje o por problemas personales relacionados con su entorno familiar, sino que también se estrellan contra un sistema educativo que no ha sido capaz de proporcionar las respuestas adecuadas a sus necesidades" (p. 138). Además, habría que considerar las mutuas relaciones e influencias entre estos factores o variables de éxito y el fracaso escolar (González, Caso, Díaz y López, 2012), lo que vendría a señalar la dificultad de abordar este problema, el abandono escolar.

Álvarez y Martínez-González (2016) subrayan que "tanto el fracaso como el abandono escolar son fruto de una progresiva desvinculación de los estudiantes de las instituciones escolares" (p. 177), entendiéndose el fracaso como la «no aprobación en el tiempo previsto -ya sea por rezago, repetición o desafiliación- en el tramo educativo que se está transitando" (Aristimuño, 2015, p. 110); sin embargo este estudio se centra en el abandono escolar prematuro, acaecido este por momentos evolutivos especialmente críticos como la adolescencia, o coincidentes con circunstancias estresantes o disfuncionales en el ámbito familiar o escolar (Álvarez y Martínez-González, 2016). Las investigaciones centradas en conocer la prevalencia del abandono escolar e identificar los factores o variables que podrían incidir en la decisión de abandonar los estudios (Alegre y Benito, 2010; Camacho, 2016; González; García, Ruíz y Muñoz, 2015), suelen tener una tendencia a mirar el abandono escolar desde una perspectiva individual, relacionándolo a la figura del alumno "fracasado", que, a pesar de ser bastante extendida, está siendo reemplazada por la perspectiva sistémica (Faubert, 2012; Fullan, 2010; González, 2005; González, 2006; Martínez, 2011, OECD, 2010;Saban- 
El liderazgo inclusivo como estrategia para evitar el abandono escolar: opinión de las familias

Emilio Crisol Moya y María Asunción Romero López

do, 2016; Sellman et al., 2002), que entiende el abandono escolar del alumnado como una consecuencia de la prestación deficiente de servicios educativos por parte de los sistemas escolares y de las escuelas, para proporcionar una educación apropiada a las necesidades del alumnado. Desde esta perspectiva, "se entiende que no solo los sistemas educativos son los responsables del problema en cuestión, sino que también implicaría a los sistemas políticos y sociales" (Sabando, 2016, p. 31). Desde este punto de vista de la educación, se estaría haciendo referencia a la participación de las familias en los centros educativos.

Aunque los factores determinantes del abandono escolar, como hemos indicado anteriormente, suelen ser multicausales (Martínez-Seijo, Rayón y Torrego, 2017), el entorno familiar en la mayoría de las ocasiones tiene un claro factor predictivo, convirtiéndose así en uno de los más influyentes (Alemany, et al., 2013; Lyche, 2013; Rumberger, 2011; Casquero y Navarro, 2010; Espínola y Claro, 2010; Mora, 2010; Roca, 2010). En la línea de nuestro objeto de estudio, algunos estudios afirman que existe una clara relación entre la colaboración formal de la familia con el centro educativo y el rendimiento del alumno (Alemany et al., 2013; Rumberger, 2011; Lyche, 2013). Es decir, desde estos trabajos se considera que es un factor decisivo la frecuencia con la que la familia contacta con el profesorado y su participación en las actividades del centro. En este sentido, para Alemany et al. (2013) y Lyche (2013) es determinante la relación con el centro educativo y la asistencia a reuniones, el seguimiento de las faltas de asistencia y la participación de las familias en el AMPA o en el Consejo Escolar. Desde esta perspectiva se considera "que si las familias apoyan a sus hijos en el estudio y participan formalmente en el centro educativo, el rendimiento escolar será mayor, disminuyendo el posible absentismo, prolongándose la vida escolar, y por tanto reduciéndose la posibilidad de abandono escolar" (Seijo, Rumayor y Torrego, 2016, p. 60).

Los resultados obtenidos en otros estudios Ilevados a cabo por Castro, Expósito, Lizasoain, López y Navarro (2014a, 2014b, 2014c) sobre la relación entre participación familiar y rendimiento, evidencian que la participación informal tiene mayor relevancia que la formal (Castro et al., 2014a); revelan como factores determinantes en el rendimiento escolar del alumnado las aspiraciones académicas de las familias, con un impacto incluso mayor que el nivel sociocultural (Castro et al., 2014b); el sentimiento de pertenencia de la familia al centro, es considerado como 
un factor que tiene una gran influencia sobre el rendimiento, ya que supone compartir el modelo educativo de la escuela (Castro et al., 2014a).

La participación de la familia puede entenderse como un recurso para la mejora de los procesos y resultados educativos; o como un recurso que permite el diseño de programas que impliquen a la familia y disminuyan las dificultades que puedan darse en esa relación, dificultades derivadas de la cultura, las creencias, la falta de confianza en los profesores, la rivalidad, la diversidad y singularidad de las familias, la diversidad de propuestas y actividades o el tipo de escuela (Calvo, Verdugo y Amor, 2016).

Los beneficios que se derivan de la participación de las familias en los centros generan efectos en los estudiantes, profesorado y en la propia familia (García-Bacete, 2003). En cuanto al alumnado, algunos de los identificados son: mejores notas; mejores puntuaciones en test de rendimiento; mayor acceso a etapas posteriores; actitudes más favorables hacia las tareas escolares; conducta más adaptativa; autoestima más elevada; realización de los deberes, tenacidad y perseverancia académica; participación en las actividades del aula; menor escolarización en programas específicos de aprendizaje; y menos tasa de abandonos y absentismo. Al igual, en el profesorado cabe destacar: los padres les reconocen mejores habilidades interpersonales y de enseñanza; los directores valoran más su desempeño docente; mayor satisfacción con su profesión; y mayor compromiso con la enseñanza. Y, en los mismos padres los efectos más destacables son: incrementan su sentido de autoeficacia; incrementan la comprensión de los programas escolares; mejoran la comunicación con sus hijos en general y sobre las tareas escolares en particular; mayor motivación para continuar su propia educación, desarrollan habilidades positivas de paternidad; y valoran más su papel en la educación de sus hijos.

\section{Método}

El objetivo y la finalidad de la investigación condicionan la metodología más adecuada (Cerda, 2011). Partiendo del carácter de este estudio se elige una metodología exploratoria y descriptiva, habitualmente utilizada en la investigación educativa. Esta permite describir un fenómeno concreto, en este caso, determinar la opinión de las familias respecto al 
El liderazgo inclusivo como estrategia para evitar el abandono escolar: opinión de las familias

Emilio Crisol Moya y María Asunción Romero López

liderazgo inclusivo desarrollo por los equipos directivos como estrategia para evitar el abandono escolar.

En este trabajo se ha optado por un enfoque metodológico de corte cuantitativo, ya que esta metodología es apropiada e idónea para abordar los objetivos del trabajo. Se trata de un estudio cuantitativo ex-post facto (Bisquerra, 2014), de tipo transversal, donde se llevó un análisis descriptivo con el apoyo del software SPSS 24.

\section{Objetivo}

El propósito general de la investigación ha sido conocer, según la opinión de las familias, la forma en que la dirección de centros de enseñanza obligatoria de Granada (España) Ileva a cabo acciones que promueven un liderazgo inclusivo, tales como los procesos de apertura del centro hacia el entorno y las familias y el centro como espacio inclusivo, que emprende acciones para impulsar la participación y atender a la diversidad, y prevenir y gestionar los conflictos en el centro, en pro de un aprendizaje para todos. En concreto, en esta ocasión, nos vamos a centrar en describir en opinión de las familias qué acciones promueve el equipo directivo como estrategia para disminuir el abandono escolar.

\section{Participantes}

El muestreo por el que se optó fue el aleatorio estratificado con un cálculo del tamaño muestral que tenía en cuenta una proporción esperada del $66 \%$ (basada en datos de pilotaje previo) y una precisión del 5\%. Estableciéndose la muestra necesaria para su significatividad en 101 centros educativos de Granada capital (37 centros privados/concertados, y 64 centros públicos). Sin embargo, aunque todos fueron invitados a responder el cuestionario, finalmente respondieron un total de 13 centros, 8 públicos y 5 privados/concertados. Por lo que al final se trata de un muestreo de carácter intencional en el que se ha tenido en cuenta que haya representación de todos los estratos.

Los miembros de las familias que han respondido el cuestionario han sido 150 , de los cuales 100 son mujeres $(66.7 \%)$ y 50 hombres $(33.3 \%)$ 
pertenecientes a centros públicos 107 (74 mujeres y 33 hombres) y 43 (26 mujeres y 17 hombres) a centros privados/concertados.

\section{Instrumento}

El instrumento "Cuestionario: Liderando la Educación Inclusiva" (LEI-Q) (Familias) (León et al., 2016) utilizado, está compuesto por 35 ítems y distribuido en dos dimensiones; fue aplicado de manera presencial en los centros educativos, tras un primer contacto telefónico con el equipo directivo. El cuestionario posee validez de contenido y fiabilidad (Alfa de Cronbach total: .981; Dimensión I: .966; Dimensión II: .963); es de tipo Likert, con cuatro opciones de respuesta (1. No implantado; 2. Parcialmente implantado; 3. Sustancialmente implantado y 4 . Totalmente implantado). El cuestionario fue aplicado durante el curso escolar 2017/18. Se optó por distribuir el cuestionario en versión papel, con la intención de aprovechar las ventajas que reporta el contacto directo a pesar de los inconvenientes derivados del coste económico y temporal.

\section{Resultados y Discusión}

A continuación, presentamos los resultados obtenidos a partir de las opiniones de las familias de los centros que decidieron participar en la investigación. Para ello hemos optado por describir cada uno de los ítems que componen las dos dimensiones: I. Apertura a la comunidad y II. El centro educativo como espacio inclusivo, que integran el "Cuestionario: Liderando la Educación Inclusiva" (LEI-Q) (León et al., 2016) dirigido a familias, a fin de hacer inferencias en cada una de sus opiniones.

Del análisis descriptivo de los datos se desprenden diversas conclusiones que contribuyen a enriquecer la literatura existente y proporcionan un punto de partida para su contraste con otras experiencias análogas.

Los resultados del análisis descriptivo por dimensiones se muestran en la Tabla 1 y Tabla 2, en las que se pueden apreciar que las medias en ambas dimensiones se encuentran entre el 3.43 y el 2.03.

El análisis por ítems de la respuesta dada por la muestra de familias de los centros participantes con respecto a las acciones más abordadas por los equipos directivos, en relación a la apertura a la comunidad (tabla 
El liderazgo inclusivo como estrategia para evitar el abandono escolar: opinión de las familias

Emilio Crisol Moya y María Asunción Romero López

1) muestra que los equipos directivos promueven acciones que evitan el fracaso escolar y se encuentran sustancialmente implantadas: propone actividades educativas fuera del centro (3.03); impulsa iniciativas que favorecen la participación de los miembros de la comunidad en el proceso formativo y en la vida del centro (3.08); promueve acciones que facilitan la comunicación y participación de todas las familias en las actividades educativas emprendidas dentro y fuera del entorno escolar (3.09); ofrece las instalaciones del centro y sus recursos para el desarrollo de actividades (culturales, educativas, etc.) de interés para la comunidad (3.11); y escucha y tiene en cuenta las demandas y necesidades de todas las familias (3.11). En estas dos últimas acciones coinciden en considerarlas las que están más implantadas, es decir, hay bastantes evidencias de que el enunciado sea cierto.

Aun así, consideran que las siguientes iniciativas están parcialmente implantadas: establecer un plan de actuaciones, elaborado en colaboración con otros miembros de la comunidad, para fomentar las relaciones centro educativo/comunidad y responder a la diversidad del alumnado (2.99); participar en las acciones emprendidas por otras instituciones/ organizaciones de la comunidad con carácter educativo (actividades deportivas, jornada contra racismo, etc.) (2.77); informar a la familia de la propuesta curricular que orienta la acción educativa del centro a través de distintos canales de comunicación $(2,95)$; impulsar acciones para sensibilizar a las familias sobre la importancia y beneficios de la inclusión $(2,78)$; establecer acciones que fomentan la representación real de la diversidad de familias existentes en los organismos de gobierno del centro (2.61); promover actividades que impulsan el conocimiento mutuo, el intercambio y la convivencia entre las familias y los demás miembros de la institución educativa (2.79); y prever medidas para contrarrestar la influencia negativa que pudiera tener la situación familiar en el éxito de sus estudiantes (campañas de ayuda, apoyos al aprendizaje, escuela de padres, programas de acompañamiento, etc.) $(2,71)$.

Son destacables, dentro de esta dimensión, aquellas acciones que las familias consideran que el equipo directivo debe promover, pues aunque están parcialmente implantadas consideran que sigue habiendo acciones que podrían mejorarse; estas se corresponden con las puntuaciones medias más bajas de la dimensión, siendo: impulsar acciones para colaborar con otros centros educativos, conocer y compartir experiencias (2.48); la colaboración con el mundo empresarial de manera continuada 
para reforzar la relación escuela-entorno laboral (2.03); y organizar debates abiertos a la comunidad sobre situaciones de exclusión (racismo, xenofobia, machismo, etc.) (2.34).

En cuanto a la desviación típica, la opinión de las familias se concentra en las acciones: promueve acciones que facilitan la comunicación y participación de todas las familias en las actividades educativas emprendidas dentro y fuera del entorno escolar y escucha y tiene en cuenta las demandas y necesidades de todas las familias; pues son las que tienen una menor desviación típica y por tanto mayor concentración en la opción de respuesta, que consideran están sustancialmente implantadas. En el lado opuesto, encontramos las referidas a: impulsa acciones para colaborar con otros centros educativos, conocer y compartir experiencias y organiza debates abiertos a la comunidad sobre situaciones de exclusión (racismo, xenofobia, machismo, etc.), que obtienen un valor elevado de la desviación típica, lo que muestra poca concentración en la opción de respuesta.

Tabla 1

Descriptivos (Media y desviación estándar): Dimensión I

DIMENSIÓN I. APERTURA HACIA LA COMUNIDAD.
EL EQUIPO DIRECTIVO...

1. Impulsa iniciativas que favorecen la participación de los miembros de la comunidad en el proceso formativo y en la vida del centro

2. Establece un plan de actuaciones, elaborado en colaboración con otros miembros de la comunidad, para fomentar las relaciones centro educativo/comunidad y responder a la diversidad del alumnado

3. Promueve la colaboración con el mundo empresarial de manera continuada para reforzar la relación escuela-entorno laboral

4. Impulsa acciones para colaborar con otros centros educativos, conocer y compartir experiencias

5. Propone actividades educativas fuera del centro

6. Organiza debates abiertos a la comunidad sobre situaciones de exclusión (racismo, xenofobia, machismo, ...)

7. Participa en las acciones emprendidas por otras instituciones/ organizaciones de la comunidad con carácter educativo (actividades deportivas, jornada contra racismo,...) 
El liderazgo inclusivo como estrategia para evitar el abandono escolar: opinión de las familias

Emilio Crisol Moya y María Asunción Romero López

\section{DIMENSIÓN I. APERTURA HACIA LA COMUNIDAD. EL EQUIPO DIRECTIVO...}

\section{D.T}

8. Ofrece las instalaciones del centro y sus recursos para el desarrollo de actividades (culturales, educativas, ...) de interés para la comunidad

9. Informa a la familia de la propuesta curricular que orienta la acción educativa del centro a través de distintos canales de comunicación

10. Impulsa acciones para sensibilizar a las familias sobre la importancia y beneficios de la inclusión

11. Promueve acciones que facilitan la comunicación y participación de todas las familias en las actividades educativas emprendidas dentro y fuera del entorno escolar

12. Establece acciones que fomentan la representación real de la diversidad de familias existentes en los organismos de gobierno del centro

13. Escucha y tiene en cuenta las demandas y necesidades de todas las familias

14. Promueve actividades que impulsan el conocimiento mutuo, el intercambio y la convivencia entre las familias y los demás miembros de la institución educativa

15. Prevé medidas para contrarrestar la influencia negativa que pudiera tener la situación familiar en el éxito de sus estudiantes (campañas de ayuda, apoyos al aprendizaje, escuela de padres, programas de acompañamiento ...)

Por lo que respecta a la dimensión del centro como comunidad inclusiva (tabla 2), son mayoritarias las acciones que en opinión de las familias están sustancialmente implantadas, siendo estas: preocuparse de que los servicios que ofrece el centro respeten las diferentes necesidades del alumnado (sensibilidades religiosas, intolerancias gastronómicas, problemas de salud, etc.) (3.31); interés por garantizar la igualdad de oportunidades movilizando recursos (materiales y humanos) con el fin de favorecer la inclusión (3.15); preocuparse por dotar al centro de recursos materiales y humanos (de profesional especializado) para promover procesos de mejora (3.05); trabajar para que exista un clima institucional en el que todo el alumnado sea reconocido, atendido y apreciado (3.25); establecer protocolos para abordar los conflictos a través del diálogo, la mediación y la negociación entre las partes implicadas (3.02); ofrecer información transparente respecto al proceso de admisión y matriculación para garantizar que llega a todos los interesados por igual (3.43); tomar medidas para prevenir y evitar 
el absentismo escolar (3.21); desarrollar programas educativos para prevenir actitudes discriminatorias entre el alumnado (3.09); generar oportunidades para que todos los miembros de la comunidad educativa participen de forma efectiva en las decisiones (3.09); promover que los distintos miembros de la comunidad educativa participen en la evaluación de las labores de dirección (3.00); establecer mecanismos para impulsar la participación del alumnado en la regulación de conflictos que surgen en el entorno educativo (3.10); y propiciar que el alumnado pueda expresar libremente su opinión y necesidades (respecto de su proceso educativo, normas y funcionamiento del centro, etc.) (3.37).

En cuanto a las acciones que consideran están parcialmente implantadas, las familias destacan: disponer de un procedimiento de recogida de información sobre las necesidades del profesorado, alumnado y resto de personal del centro (2.48); trabajar para que exista un clima institucional en el que todo el alumnado sea reconocido, atendido y apreciado (2.83); establecer sanciones por el uso de símbolos y acciones que promueven la exclusión (2.85); compartir la autoridad y responsabilidad con el profesorado (2.81); establecer procedimientos con el fin de promover la mejora del centro (2.87); disponer de mecanismos para documentar los cambios y mejoras emprendidas (2.61); promover acciones de acogida para todo el alumnado y para el profesorado de nueva incorporación (2.75); y promover la participación del alumnado en los órganos de gobierno del centro (2.90).

De los datos de la desviación típica, podemos destacar las afirmaciones obtienen las puntuaciones más bajas, lo que confirma la concentración de las opiniones de las familias en estas, considerándolas sustancialmente implantadas; siendo: se preocupa de que los servicios que ofrece el centro respeten las diferentes necesidades del alumnado, ofrece información transparente respecto al proceso de admisión y matriculación para garantizar que llega a todos los interesados por igual y propicia que el alumnado pueda expresar libremente su opinión y necesidades. En el caso contrario, las puntuaciones más altas coinciden en las afirmaciones: establece protocolos para abordar los conflictos a través del diálogo, la mediación y la negociación entre las partes implicadas, dispone de mecanismos para documentar los cambios y mejoras emprendidas y el equipo directivo promueve acciones de acogida para todo el alumnado y para el profesorado de nueva incorporación; mostrando la falta acuerdo en la opinión de las familias respecto de estas. 
El liderazgo inclusivo como estrategia para evitar el abandono escolar: opinión de las familias

Emilio Crisol Moya y María Asunción Romero López

Tabla 2

Descriptivos (Media y desviación estándar): Dimensión II

\section{DIMENSIÓN II. EL CENTRO COMO COMUNIDAD IN- CLUSIVA.}

EL EQUIPO DIRECTIVO...

M D.T

16. Dispone de un procedimiento de recogida de información sobre las necesidades del profesorado, alumnado y resto de personal del centro

17. Se preocupa de que los servicios que ofrece el centro respeten las diferentes necesidades del alumnado (sensibilidades religiosas, intolerancias gastronómicas, problemas de salud, ...)

18. Se interesa por garantizar la igualdad de oportunidades movilizando recursos (materiales y humanos) con el fin de favorecer la inclusión

19. Se preocupa por dotar al centro de recursos materiales y humanos (de profesional especializado) para promover procesos de mejora

20. Trabaja para que exista un clima institucional en el que todo el alumnado sea reconocido, atendido y apreciado

21. Promueve entre el profesorado una visión compartida sobre la organización, metas y actividades para hacerles partícipes de un proyecto común

22. Establece protocolos para abordar los conflictos a través del diálogo, la mediación y la negociación entre las partes implicadas

23. Ofrece información transparente respecto al proceso de admisión y matriculación para garantizar que llega a todos los interesados por igual

24. Toma medidas para prevenir y evitar el absentismo escolar

25. Establece sanciones por el uso de símbolos y acciones que promueven la exclusión

26. Desarrolla programas educativos para prevenir actitudes discriminatorias entre el alumnado

27. Comparte la autoridad y responsabilidad con el profesorado

28. Genera oportunidades para que todos los miembros de la comunidad educativa participen de forma efectiva en las decisiones

29. Establece procedimientos con el fin de promover la mejora del centro

30. Dispone de mecanismos para documentar los cambios y mejoras emprendidas 


\section{DIMENSIÓN II. EL CENTRO COMO COMUNIDAD IN- CLUSIVA.}

EL EQUIPO DIRECTIVO...

M D.T

31. Promueve que los distintos miembros de la comunidad educativa participen en la evaluación de las labores de dirección 3.001 .03

32. El equipo directivo promueve acciones de acogida para todo el alumnado y para el profesorado de nueva incorporación

33. Promueve la participación del alumnado en los órganos de gobierno del centro

34. Establece mecanismos para impulsar la participación del alumnado en la regulación de conflictos que surgen en el entorno educativo

35. Propicia que el alumnado pueda expresar libremente su opinión y necesidades (respecto de su proceso educativo, normas y funcionamiento del centro, ...)

\section{Conclusiones}

Los resultados permiten identificar fortalezas y debilidades de las acciones emprendidas por los equipos directivos de los centros de Granada. De manera general, para el total de la muestra de las familias, las acciones que contribuyen a la disminución del abandono escolar y que el equipo directivo promueve dentro del liderazgo inclusivo, son las relacionadas con tres de los factores que algunas investigaciones (De Witte, et al., 2014) identifican como claves en la intervención del abandono escolar: Ios familiares, de escuela y de la comunidad.

Concretamente, de la primera dimensión, destacan las acciones referidas a la apertura hacia el entorno y la comunidad para promover la participación de estos en el proceso formativo y en la vida del centro; y, en relación a la familia, las acciones que facilitan la comunicación y participación (atendiendo sus demandas y necesidades) de las actividades dentro y fuera del entorno escolar.

Desde la literatura se considera un factor decisivo para prevenir el abandono escolar la frecuencia con la que la familia contacta con el profesorado y si participa en las actividades del centro (Alemany et al., 2013; Lyche, 2013; Rumberger, 2011). Es determinante la relación con el centro educativo y la asistencia a reuniones, el seguimiento de las faltas de asistencia y la participación de las familias en Escuelas de Padres, en el AMPA o en el Consejo Escolar (Alemany et al., 2013 y Lyche, 2013). 
De las acciones que hay que seguir trabajando para mejorar, destacan las relacionadas con la colaboración con el mundo empresarial, como en otras investigaciones (Pàmies, Senent y Essomba, 2016), la organización de debates abiertos a la comunidad sobre situaciones de exclusión (racismo, xenofobia, machismo, etc.), así como la colaboración con otros centros educativos, para conocer y compartir experiencias.

De la segunda dimensión, el centro como comunidad inclusiva, se señalan aquellas acciones que generan una visión compartida, promoviendo la participación, cooperación y dinámicas de reflexión positiva hacia la diversidad; pues la formación y participación decisiva de las familias en los centros educativos disminuyen el posible absentismo y prolonga la vida escolar (Álvarez y Martínez, 2016).

Concretamente, en nuestra investigación, destacan ofrecer información transparente respecto al proceso de admisión y matriculación para garantizar que llegue a todos los interesados por igual; propiciar que el alumnado pueda expresar libremente su opinión y necesidades (respecto de su proceso educativo, normas y funcionamiento del centro, etc.); preocuparse de que los servicios que ofrece el centro respeten las diferentes necesidades del alumnado (sensibilidades religiosas, intolerancias gastronómicas, problemas de salud, etc.); y, tomar medidas para prevenir y evitar el absentismo escolar, esta última acción directamente relacionada con el abandono escolar.

Dar una respuesta adecuada a las necesidades de los estudiantes es un elemento clave para que las escuelas afronten el abandono escolar (Rittaco y Amores, 2016).

De esta segunda dimensión, se considera necesario atender aquellas acciones que podrían mejorar, como disponer de un procedimiento de recogida de información sobre las necesidades del profesorado, alumnado y resto de personal del centro, así como de mecanismos para documentar los cambios y mejoras emprendidas.

Como señalan Reparaz y Naval (2014), parece esencial promover cauces de formación comunitaria conjunta como modelo que favorece y promueve la participación en todas sus modalidades: información, toma de decisiones, planificación, desarrollo y evaluación de la tarea educativa; diseñada colaborativamente por familias y docentes.

En esta misma línea, Vallespir, Rincón y Morey (2016) proponen un formación bicéfala, en ambos sentidos, en la que las familias reciben 
una formación planificada, estructurada, implementada, y evaluada, contemplada en el ideario del centro educativo y, en el caso de los docentes que abarque tanto la formación inicial como continua, centrada en contenidos como: canales de comunicación, atención individualizada y grupal, tipologías y estrategias de participación, técnicas de dinamización de grupos y educación de adultos.

Marchesi y Hernández (2003, p. 40), destacan que factores como "(...) el liderazgo del equipo directivo, el ambiente favorable de aprendizaje, la existencia de un proyecto compartido, la organización eficiente de la enseñanza en el aula, la participación de los padres y de los alumnos, el seguimiento del progreso de los alumnos y la evaluación de las escuelas", contribuyan a la prevención del abandono escolar.

\section{Referencias}

Ainscow, M. y Kaplan, I. (2005). Usingevidencetoencourage inclusive schooldevelopment: Possibilities and challenges. AustralasianJournal of SpecialEducation, 29(2), 106-116. doi.org/10.1080/1030011050290203

Ainscow, M., Both, T., y Dyson, A. (2006). Inclusion and thestandards agenda: negotiatingpolicypressures in England. International journal of inclusive education, 10(4-5), 295-308.

Alegre, M. A., y Benito, R. (2010). Los factores del abandono educativo temprano. España en el marco europeo. Revista de Educación, Número Extraordinario, 6592.

Álvarez, B., y Martínez-González, R. (2016). Cooperación entre las Familias y los Centros Escolares como Medida Preventiva del Fracaso y del Riesgo de Abandono Escolar en Adolescentes. Revista Latinoamericana de Educación Inclusiva, 2016, 10 (1), 175192.

Alemany, I., Rojas, R., Gallardo, M. I., y Sánchez, S. (2013). El abandono escolar temprano en un contexto multicultural. Análisis de sus causas por los agentes profesionales y sociales implicados. JournalforEducators, Teachers and Trainers, 4(2), 191-203.

Álvarez Blanco, L. y Martínez-González, R. A. (2016). Cooperación entre las familias y los centros escolares como medida preventiva del fracaso y del riesgo de abandono escolar en adolescentes. Revista latinoamericana de educación inclusiva, 10(1), 175192.

Angelides, P., Antoniou, E., y Charalambous, C. (2010). Makingsense of inclusión for leadership and schooling: A case study from Cyprus. International Journal of Leadership in Education, 13(3), 319-334. doi.org/10.1080/13603120902759539

Aristimuño, A. (2015). El fracaso escolar, ¿fracaso de quién? La modificación del concepto de fracaso. Revista Latinoamericana Educación Inclusiva, 9(1), 111-126. 
El liderazgo inclusivo como estrategia para evitar el abandono escolar: opinión de las familias

Emilio Crisol Moya y María Asunción Romero López

Batanova, M. y Loukas, A. (2014). Unique and interactiveeffects of empathy, family, and schoolfactorsonearlyadolescents' aggression. Journal of Youth and Adolescence, 43(11), 1.890-1.902.

Bisquerra, R. (Coord.) (2014). Metodología de la investigación educativa (4a ed.). La Muralla.

Bolívar, A. (2010). ¿Cómo un liderazgo pedagógico y distribuido mejora los logros académicos? Revisión de la investigación y propuesta. MAGIS, 3(5), 79-106.

Bolívar, A., López, J. y Murillo, F.J. (2013). Liderazgo en las instituciones educativas. Una revisión de líneas de investigación. Revista Fuentes, 14, 15-60.

Booth, T. y Ainscow, M. (2000). Guía para la evaluación y la mejora de la educación inclusiva. Desarrollando el aprendizaje y la participación en las escuelas. Universidad autónoma de Madrid.

Calvo, M. I, Verdugo, M. A, \& Amor, A. M. (2016). La Participación Familiar es un Requisito Imprescindible para una Escuela Inclusiva. Revista latinoamericana de educación inclusiva, 10(1), 99-113. doi.org/10.4067/S0718-73782016000100006

Camacho, M. A. (2016). Fracaso escolar y abandono educativo temprano en Educación Secundaria Obligatoria: un estudio integrado. Tesis Doctoral. Universidad de Huelva.

Casquero, A., y Navarro, M. L. (2010). Determinantes del abandono escolar temprano en España: un análisis por género. Revista de Educación, Número Extraordinario, 191-223.

Castro, M., Expósito, E., Lizasoain, L., López, E., y Navarro, E. (2014a). Participación familiar y rendimiento académico. Una síntesis meta-analítica. Ministerio de Educación, Cultura y Deporte. La participación de las familias en la Educación Escolar (pp. 83-105). Madrid, España: Secretaría General Técnica.

Castro, M., Expósito, E., Lizasoain, L., López, E., y Navarro, E. (2014b). Participación familiar y rendimiento académico de alumnos españoles de Educación Infantil, Educación Primaria y Educación Secundaria Obligatoria. Ministerio de Educación, Cultura y Deporte. La participación de las familias en la Educación Escolar (pp. 167-179). Madrid, España: Secretaría General Técnica.

Castro, M., Expósito, E., Lizasoain, L., López, E., y Navarro, E. (2014c). Evaluación del impacto de la participación familiar sobre la competencia matemática en PISA 2012. Un estudio internacional comparado. Ministerio de Educación, Cultura y Deporte. La participación de las familias en la Educación Escolar (pp. 107-125). Madrid, España: Secretaría General Técnica.

Cerda, H. (2011). Los elementos de la investigación: cómo reconocerlos, diseñarlos y construirlos. Editorial Magisterio.

De Witte, K., Cabus, S., Thyssen, G.,Groot, W., y Brink, H. M. (2014). A Critical Review of the Literature on School Dropout. Tier Working Paper Series.

Echeita, G. (2012). Competencias esenciales en la formación inicial de un profesorado inclusivo. Un proyecto de la agencia europea para el desarrollo de las necesidades educativas especiales. Tendencias pedagógicas, 19, 7-24.

Escudero, J. M. y Martínez, B. (2011). Educación inclusiva y cambio escolar. Revista Iberoamericana de Educación, 55, 85-105. 
El liderazgo inclusivo como estrategia para evitar el abandono escolar: opinión de las

familias

Emilio Crisol Moya y María Asunción Romero López

Espínola, V., y Claro, J. P. (2010). Estrategias de prevención de la deserción en la Educación Secundaria: perspectiva latinoamericana. Revista de Educación, Número Extraordinario, 257-280.

Faubert, B. (2012). A literatura review of schoolpracticestoovercomeschoolfailure, OECD Educationworkingpapers, 68, OECD Publishing. doi. org $/ 10.1787 / 5 \mathrm{kgflcwwv} 9 \mathrm{tk}$-en

Fullan, M. (2010). Allsystemsgo: Thechangeimperativeforwholesystemreform. Corwin.

García-Bacete, F.J. (2003). Las relaciones escuela-familia: un reto educativo", Infancia y Aprendizaje: JournalfortheStudy of Education and Development, 26 (4), 425- 437. doi.org/10.1174/021037003322553824

González, C., Caso, J., Díaz, K., y López, M. (2012). Rendimiento académico y factores asociados. Aportaciones de algunas evaluaciones a gran escala. Bordón, Revista dePedagogía, 64 (2), 51-68.

González, M. T. (2005). El absentismo y el abandono. Una forma de exclusión escolar. Profesorado. Revista de currículum y formación del profesorado, 9(1), 1-12.

González, M. T. (2006). Absentismo y abandono escolar: una situación singular de exclusión educativa. REICE. Revista Iberoamericana sobre Calidad, Eficacia y Cambio en Educación, 4(1), 1-15.

González, S., García, M- P. Ruiz, F., y Muñoz, J. M. (2015). Factores de riesgo del abandono escolar desde la perspectiva del profesorado de Educación Secundaria Obligatoria. Profesorado. Revista de currículum y formación del profesorado, 19, 226-245. Recuperado de https://recyt.fecyt.es/index.php/profesorado/article/view/43646

León, M. J. (2011). La situación de la formación en educación inclusiva en los nuevos títulos de grado de maestro enEspaña. Revista interuniversitaria de formación del profesorado, 70, 145-163.

León, M. J. (2012). El liderazgo para y en la escuela inclusiva. Educatio Siglo XXI, 30(1), 133-160.

León, M. J., Crisol, E., y Moreno, R. (2018). Las Tareas del Líder Inclusivo en Centros Educativos de Zonas Desfavorecidas y Favorecidas.REICE. Revista Iberoamericana sobre Calidad, Eficacia y Cambio en Educación, 16(2), 21-40. doi.org/10.15366/reice2018.16.2.002

León, M. J., López, M. C., Romero, A., Crisol, E., Hinojosa, E., y Moreno, R. (2016). Liderando la educación inclusiva en centros de educación primaria y secundaria. En J.L. Bernal (Coord.), XIV Congreso interuniversitario de organización de instituciones educativas (CIOIE). Liderazgo inclusivo y para la justicia social. Congreso llevado a cabo en Zaragoza, España.

Lozano, A. (2012). Factores personales, familiares y académicos que afectan al fracaso escolar en la Educación Secundaria. Revista Electrónica de Investigación Psicoeducativa y Psicopedagógica, 1 (1), 43-66.

Lyche, C. (2013). Taking on the Completion Challenge: A Literature Review on Policies to Prevent Drop out and Early School Leaving. OECD Education Working Papers, $\mathrm{n}^{\circ} 53$, OECD Publishing. doi: 10.1787 /5km4m2t59cmr-en.

Marchesi, A., y Hernández, C. (2003). El fracaso escolar: una perspectiva internacional. Alianza Estudio. 
El liderazgo inclusivo como estrategia para evitar el abandono escolar: opinión de las familias

Emilio Crisol Moya y María Asunción Romero López

Martínez-Seijo,M., Rayón, L., y Torrego J.C.(2017). Las familias ante el abandono escolar. Bordón, Revista de Pedagogía, 69 (2), 59-78.

Martínez, B. (2011). Luces y sombras de las medidas de atención a la diversidad en el camino de la inclusión educativa. Revista Interuniversitaria de Formación del Profesorado, 25(1), 165-183.

Mora, A. J. (2010). Determinantes del abandono escolar en Cataluña: más allá del nivel socio-económico de las familias. Revista de Educación, Número Extraordinario, 171190.

Moreno, R. y León, M. J. (2017). Análisis del liderazgo inclusivo en centros educativos de Primaria y Secundaria de Granada. ReiDoCrea, 6, 211-220.

Muijs, D. (2006). New directions for school effective eness research: Towards school effective nes swithout schools. Journal of Educational Change, 7(3), 141-160. doi. org/10.1007/s10833-006-0002-7

OrganisationforEconomic Co-operation and Development. (2010). Overcomingfailure at school: Policiesthatwork. OECD Project Description. Paris: OECD Publishing. Recuperado de www.oecd.org/dataoecd/54/54/45171670.pdf

OrganisationforEconomic Co-operation and Development. (2018). Informe español. Informe elaborado por el Instituto Nacional de Evaluación Educativa (INEE) y que reproduce datos de los indicadores más relevantes para España en comparación con la media de los países de la OCDE.

Pàmies-Rovira, J., Senent-Sánchez, J. M., \&Essomba-Gelabert, M. A. (2016). El liderazgo pedagógico y la implicación del profesorado como factores de éxito en centros de entornos desfavorecidos en España. RELIEVE, 22(2). doi.org/10.7203/relieve.22.2.7600

Reparaz, C., y Naval, C. (2014). Bases conceptuales de la participación de las familias. En Consejo Escolar del Estado, La participación de las familias en la educación escolar (pp. 21-34). Madrid: Subdirección General de Documentación y Publicaciones. Recuperado de http://ntic.educacion.es/cee/estudioparticipacion

Ritacco, M. y Amores, F. J. (2016). Estudiantes en riesgo de exclusión educativa en secundaria. Percepciones del profesorado implicado en programas extraordinarios. Enseñanza \& Teaching, 34 (1), 137-160.

Roberts, G. (2018). Respuestas del sector de la educación frente al consumo de alcohol, tabaco y drogas (Vol. 10). UNESCO Publishing.

Roca, E., (2010). El abandono temprano de la educación y la formación en España. Revista de Educación, Número Extraordinario, 31-62.

Rumberger, R. (2011). From Dropping Out. Why stundents droppout High School and What can be done about it. Boston, EE. UU.: Harvard University Press.

Ryan, J. (2006). Inclusive leadership and social justice for schools. Leadership and Policy in Schools, 5(1), 3-17 doi.org/10.1080/15700760500483995

Sabando, D. S. (2016). Inclusión educativa y rendimiento académico. Relación entre el Grado de Inclusión y el Rendimiento Académico en las Escuelas Públicas de Primaria de Cataluña. Tesis doctoral. Universidad de Barcelona, Barcelona.

Simón, C., Giné, C., y Echeita, G. (2016). Escuela, Familia y Comunidad: Construyendo Alianzas para Promover la Inclusión. Revista Latinoamericana de Educación Inclusiva, 10 (1). doi.org/10.4067/S0718-73782016000100006. 
El liderazgo inclusivo como estrategia para evitar el abandono escolar: opinión de las

familias

Emilio Crisol Moya y María Asunción Romero López

Sellman, E., Bedward, J., Cole, T., y Daniels, H. (2002). Thematic review: A sociocultural approach to exclusion. British Educational Research Journal, 28(6), 889-900.

Vallespir, J., Rincón, J.C., y Morey, M. (2017). La documentación oficial de los centros y la participación de las familias. En Garreta, J. (Coord.) (2017). Familias y escuelas. Discursos y prácticas sobre la participación en la escuela. Madrid: Pirámide. pp: 49-69. 
